\title{
Collisional Focusing Effects in Radio Frequency Quadrupoles
}

\author{
D. J. Douglas \\ SCIEX, Thornhill, Ontario, Canada
}

J. B. French

University of Toronto Institute for Aerospace Studies, Downsview, Ontario, Canada

The transmission of ions through a conventional two-dimensional radiofrequency-only ( $\mathrm{r}$ ) quadrupole has been studied for comparatively high operating pressures between $5 \times 10^{-4}$ and $1 \times 10^{-2}$ torr. Measurements of signals from mass-resolved analyte ions and total ion currents show that, provided the initial injection ion energy is low $(1-30 \mathrm{eV})$, the ion transmission observed through a small aperture at the exit of the rf quadrupole first increases as the gas pressure increases, reaching a maximum at $-8 \times 10^{-3}$ torr before decreasing at higher pressures. This is in direct contrast to the expectations of classical scattering. This "collisional focusing" appears to be analogous to effects seen in threedimensional ion traps. The collisional focusing increases with the mass of the ion (not mass-to-charge ratio) for masses up to at least $16,950 \mathrm{u}$. The collisional focusing of the ions is found to be accompanied by significant losses of axial kinetic energy. A Monte Carlo simulation of the energy loss process is reported that can provide agreement with the observed losses for reasonable collision cross-sections. The results suggest that operation of rf quadrupoles at relatively high pressure may find practical application in sampling ions from high (e.g., atmospheric) pressure ion sources. (I Am Soc Mass Spectrom 1992, 3, $398-408)$

$\mathrm{R}$ adiofrequency-only (rf) quadrupoles have been used in several instruments to sample ions from "gassy" ion sources (often the interface to an atmospheric pressure ion source [1-3]). Ions are transported down the if quadrupole to a mass analyzing quadrupole while gas from the source is pumped away through the rods. Although scattering loss cross-sections in if quadrupoles are quite small (a few square Angstroms) [4] in comparison with gas kinetic collision cross-sections $\left(-50 \AA^{2}\right)$, some losses of ions might be expected, decreasing the ion transmission at high pressures. The work to be reported here was initially directed toward measuring these scattering loss cross-sections. Large, multiply charged ions formed by electrospray or ionspray were of particular interest. It was found, however, that for all of the ions studied (singly and multiply charged), the ion transmission increased as the gas pressure in the $\mathrm{rf}$ quadrupole increased, in direct contrast to the expectations of conventional scattering theory. The increased ion transmission was found to be accompanied by substantial changes in the axial kinetic energy distributions. The results appear to be analogous to

Address reprint requests to D. J. Douglas, SCIEX, 55 Glen Cameron Road, Thornhill, Ontario, Canada L3T 1P2. the collisional damping or collisional focusing of ion trajectories well known in three-dimensional ion traps (see, e.g., refs 5-7). To our knowledge this is the frrst report of such effects in two-dimensional rf quadrupoles. In this article our observations of these effects are described, along with a simple Monte Carlo model for the axial energy distributions.

\section{Experimental}

The apparatus used is shown schematically in Figure 1. Ions were sampled from an atmospheric pressure ion (API) source (either corona discharge [1] or ionspray [8]) into a region containing an $\mathrm{rf}$ quadrupole, pumped by a $700 \mathrm{~L} \mathrm{~s}^{-1}$ diffusion pump (Alcatel 9160 M). Ions passed through a small (either 1.0 or 2.5 $\mathrm{mm}$ ) aperture (the "interquad" aperture) into a mass analyzing quadrupole. This aperture acted as both a crude ion lens and as the differential pumping aperture between the quadrupole chambers. The analyzing quadrupole region was pumped by a $300 \mathrm{~L} \mathrm{~s}^{-1}$ diffusion pump (Alcatel $9100 \mathrm{M}$ ), The diffusion pumps were backed by a single rotary vane pump (either Leybold Heracus D60A, $17 \mathrm{~L} \mathrm{~s}^{-1}$ or D16A, 6.7 $\mathrm{L} \mathrm{s}^{-1}$ ). The pressure in the analyzing quadrupole varied from 


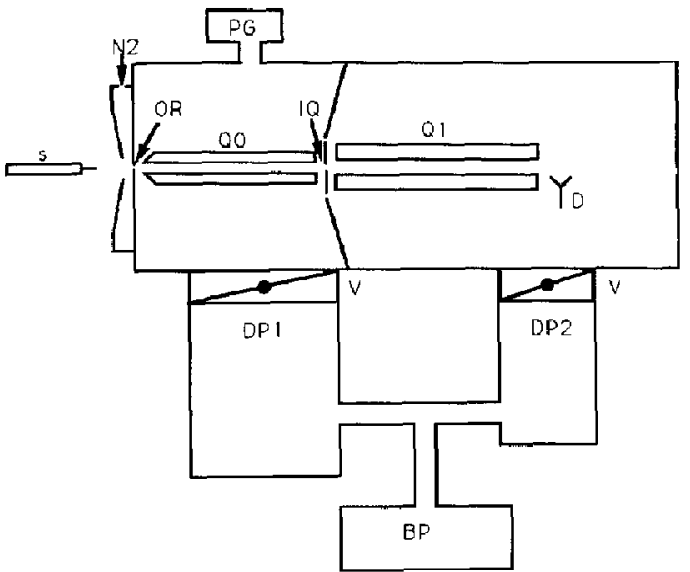

Figure 1. Schematic of the apparatus. $S=$ ion source; $N_{2}=$ nitrogen "curtain" gas; $\mathrm{OR}=$ sampling orifice; $\mathrm{QO}=$ radiofrequency-only quadrupole; $Q 1=$ mass analyzing quadrupole; IQ $=$ "interquad" aperture; $\mathbf{D}=$ detector; $\mathrm{PG}=$ capacitance manometer pressure gauge; DP1, DP2 = diffusion pumps; $\mathrm{V}=$ butterfly valves; $\mathrm{BP}=$ backing pump.

$5 \times 10^{-6}$ to $3 \times 10^{-5}$ torr, depending on the operating conditions. Butterfly valves between the vacuum chamber and diffusion pumps could be partially closed to increase the pressure either in the analyzing quadrupole region or (for most experiments) in the rf quadrupole region. The pressure in the rf quadrupole region was measured with a capacitance manometer (Baratron model 222, 1.0 torr full scale, M.K.S. Instruments, Andover, MA). Provided care was taken to correct for long-term zero drift, pressures as low as $1 \times 10^{-4}$ torr could be measured with this gauge. Ion counting was used and spectra were collected in a multichannel scaler (either ACE MCS board, E.G. and G. Ortec, Oak Ridge, TN, used with an Epson Equity III + computer, or TN7200 multichannel analyzer with a model TN1251 ramp generator, Tracor Northern, Middletown, WI, USA). The multichannel analyzer generated a ramp voltage that was used to mass scan the mass-to-charge ratio value selected by the quadrupole power supply.

The rf-only quadrupole rods were $150-\mathrm{mm}$ long with a diameter of $12.4 \mathrm{~mm}$. The analyzing quadrupole rods were $15.6 \mathrm{~mm}$ in diameter. Thus, the interquad aperture diameter was small compared to the field diameter of the quadrupoles. The voltage on the if quadrupole was derived from the main quadrupole supply through coupling capacitors which, along with the capacitance to ground of the if rods, formed an rf voltage divider. By changing the coupling capacitors or by increasing the capacitance of the rf rods to ground (with additional bypass capacitors) the voltage on the if rods and hence the Mathieu parameter " $q$ " [9] of ions in the rods could be changed. Because the voltage on the rf rods was a constant fraction of the voltage on the analyzing quadrupole, all analyte ions had the same $q$ in the $r f$ rods when the analyzing quadrupole was set to their corresponding mass. The dc rod offset voltage of the rf quadrupole was set separately from a dc supply through bias resistors to the rods. The quadrupoles were operated at a frequency of $1.0 \mathrm{MHz}$.

Two different interfaces were used with the API sources. The first, shown in Figure 1, sampled ions from atmosphere through a flat $0.089-\mathrm{mm}$ diameter orifice. A counter-flow of high purity nitrogen ("curtain gas") covered the orifice. Ions were drifted through the curtain gas with electric fields [1, 8]. The nitrogen curtain gas expanded through the orifice to form a free jet. The distance from the orifice to the entrance to the $\mathrm{rf}$ quadrupole was $9 \mathrm{~mm}$. With this interface the gas flow (nitrogen) through the orifice was 0.93 torr $\mathrm{L} \mathrm{s}^{-1}$ and the lowest pressure possible in the rf quadrupole chamber was $2.4 \times 10^{-3}$ torr (corresponding to an effective pumping speed of $\sim 400 \mathrm{~L} \mathrm{~s}^{-1}$ ). Ions could be declustered or fragmented by changing the dc voltage between the orifice and rf quadrupole.

The second interface is sketched in Figure 2. This interface used differential pumping. Ions and gas expanded through an orifice (0.25- $\mathrm{mm}$ diameter) to form a free jet, and a fraction of this flow passed through a skimmer into the region containing the rf quadrupole. Typically, the skimmer orifice was $0.75 \mathrm{~mm}$ in diameler with the skimmer tip $3 \mathrm{~mm}$ downstream of the sampling orifice, although other values for these parameters were sometimes used. A nitrogen curtain gas was used with this interface as well. The interface was pumped by a $9 \mathrm{~L} \mathrm{~s}^{-1}$ rotary vane pump (Leybold Heraeus D25A) to a pressure of $\sim .2$ torr. With this interface the gas flow into the rf rods region was lower $\left(-0.17\right.$ torr $\left.L s^{-1}\right)$ and lower pressures of $5 \times 10^{-4}$ torr could be achieved (for the skimmer orifice diameter and spacing cited). A discussion of

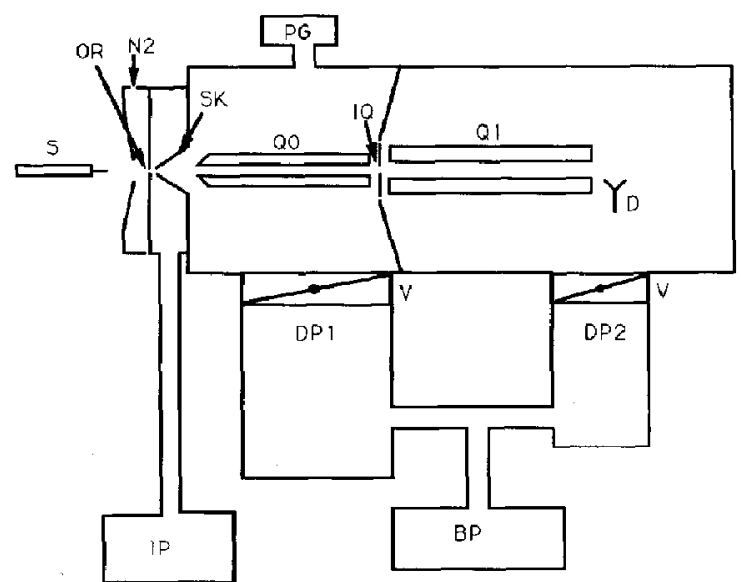

Figure 2. Schematic of the apparatus with the differentially pumped interface installed. $\mathrm{SK}=$ skimmer; $I P=$ interface vacuum pump. The other components are labeled as in Figure 1. 
the development of this interface is outside the scope of this article. Ions could be declustered or fragmented by changing the voltage between the orifice and skimmer $[10,11]$. The differentially pumped interface provided a small $(-\times 2-\times 4)$ improvement in the ratio of ions-to-gas entering the rf quadrupole. The distance from the skimmer tip to the entrance to the rf quadrupole was $45 \mathrm{~mm}$, considerably greater than with the single aperture interface. Because nitrogen was used as the curtain gas, nitrogen was the collision gas in the rf quadrupole in all experiments. It is expected however that collisional focusing could be observed with other gases (e.g., $\mathrm{He}$, Ar).

With the corona source, reagents [dimethylmorpholinophosphoramidate (DMMPA) or tris-perfluoro-heptyl-s-triazine] were introduced simply by passing a flow of carrier air $\left(-2 \mathrm{~L} \mathrm{~min}^{-1}\right)$ over an open vial of the compound and into the source [1]. DMMPA gave a protonated parent ion peak at $m / z$ 196 and a protonated dimer ion at $\mathrm{m} / z$ 391. Tris-perfluoro-heptyl-s-triazine produced a negative parent ion at $m / z 1185$ and a fragment at $m / z$ 832. Solutions of mellitin (average molecular weight 2846.4) (Sigma

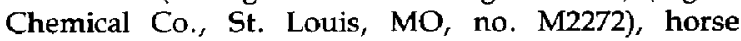
skeletal myoglobin (average molecular weight 16,950.4) (Sigma no. M0603), and bovine insulin (average molecular weight 5790) (Sigma I 5500) were prepared and infused into the ionspray source as in ref 12 and produced similar spectra. Polypropylene glycol (PPG) (average molecular weight 1000) was infused at a concentration of $10^{-4} \mathrm{M}$ in a solution of $10^{-3} \mathrm{M}$ ammonium acetate and 50:50 methanol:water to give a number of peaks in the $\mathrm{m} / \mathrm{z} 300$ to 1500 range.

Ion currents to the if quadrupole were measured by disconnecting the quadrupole power supply, connecting the four rods together electrically, and measuring the current from the rods to ground. This current was taken as the total ion current entering the rf quadrupole region. Ion currents to the interquad aperture were measured by disconnecting the lens supply and measuring the current from the aperture plate to ground.

Typical operating voltages for positive ion spectra are shown in Table 1. (For negative ion spectra the polarities were reversed.) The difference in voltage between the orifice and if quadrupole rod offset (Figure 1 apparatus) or the skimmer and rf quadrupole rod offset (Figure 2 apparatus) was taken as the nominal injection ion energy. As discussed below, with the single aperture interface collisions of ions with the relatively high density gas in the free jet can reduce the injection energies somewhat below this nominal value.

\section{Results with the Single Aperture Interface}

Our first observations of collisional focusing effects were obtained with the apparatus of Figure 1. Figure
Table 1. Voltages used for these experiments

\begin{tabular}{lcc}
\hline & $\begin{array}{c}\text { With the } \\
\text { single aperture } \\
\text { interface }\end{array}$ & $\begin{array}{c}\text { Differentially } \\
\text { pumped } \\
\text { interface }\end{array}$ \\
\hline \hline $\begin{array}{l}\text { Gas curtain plate } \\
\begin{array}{l}\text { Orifice } \\
\text { Skimmer }\end{array}\end{array}$ & +600 & +1000 \\
$\begin{array}{l}\text { Radiofrequency-only } \\
\text { quadrupole } \\
\text { offset }\end{array}$ & +25 & +150 to 200 \\
$\begin{array}{l}\text { Interquad aperture } \\
\begin{array}{l}\text { Analyzing rods offset } \\
\text { voltage }\end{array}\end{array}$ & +15 & +90 \\
\hline
\end{tabular}

3a shows the variation in transmitted ion signal for $\mathrm{m} / \mathrm{z} 196^{+}, 391^{+}$, and $832^{-}$for pressures in the if quadrupole from $2.4 \times 10^{-3}$ torr to $\sim 1.0 \times 10^{-2}$ torr for a nominal injection energy of $10 \mathrm{eV}$ and a $\mathrm{q}$ of 0.65. A $2.5-\mathrm{mm}$ interquad aperture was used. Figure $3 \mathrm{~b}$ shows similar results at a $\mathrm{q}$ of 0.19 . The relative count rate is normalized to 1.0 at a pressure of $2.4 \times$ $10^{-3}$ torr (the lowest possible with this interface). In Figure $3 a$ and $b$ it is seen that the ion signals first increase as the pressure in the rf rods increases before showing a decrease at higher pressures. This increase
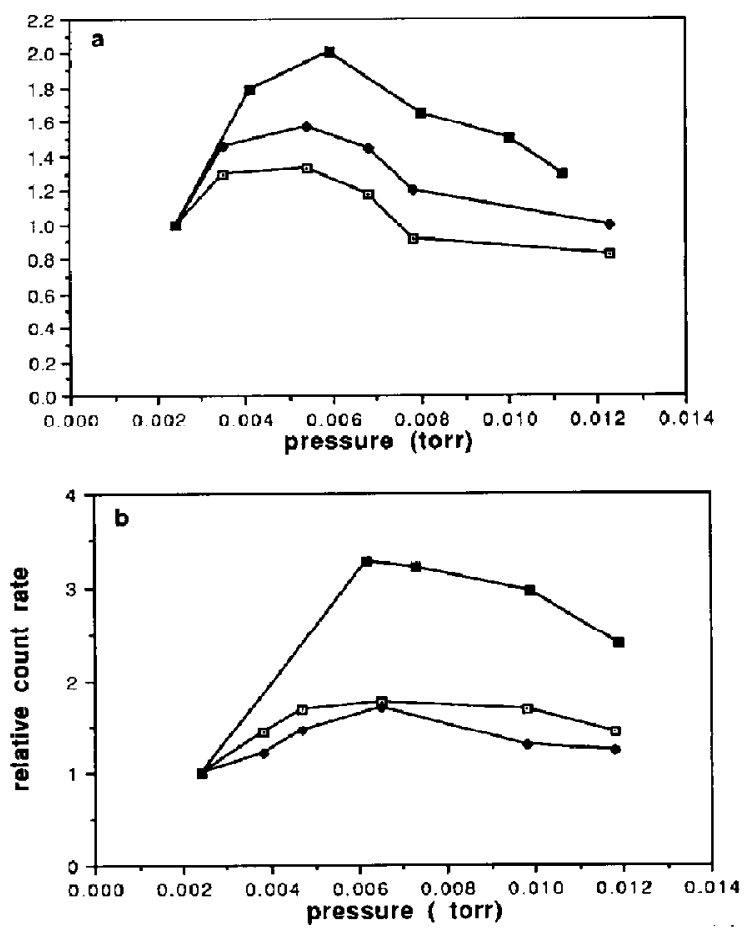

Figure 3. The variation of relative count rate for ions of ( $\square$ ) $m / z 196^{+},(\bullet) 391^{+}$, and (口) $832^{-}$with pressure in the $\mathrm{rf}$ quadrupole. The results are normalized to 1.0 at a pressure of $2.4 \times 10^{-3}$ torr. In (a) the Mathieu parameter of the ions (q) was 0.6 ; in (b) $q$ was 0.19 . 
was surprising because even a small scattering loss cross-section $\left(3 \AA^{2}\right)$, such as has been reported for if quadrupole collision celis [4], is calculated to give substantial losses in ion transmission over this pressure range (up to $-80 \%$ at $10^{-2}$ torr). Initially it was thought that this unexpected increase might be due to collision-activated dissociation (CAD) of higher mass water clusters of these ions in the relatively high pressure quadrupole. No such clusters were seen in the mass spectra, however, and repeating the experiment with a range of declustering potentials between the interface and rf rods gave essentially the same result. The increase in count rate for $m / z 196^{+}$from DMMPA might be explained by CAD of the dimer ion $m / z 391^{+}$. However, an experiment was run under strongly declustering conditions so that the dimer $m / z 391^{+}$was only $-1 \%$ of the monomer $m / z 196^{+}$. In this case the monomer $m / z 196^{+}$still showed an $-20 \%$ increase in signal that could not be accounted for even by complete conversion of the dimer to monomer (which was not seen). When the pressure in the if quadrupole region was increased to $\sim 1 \times$ $10^{-2}$ torr, the pressure in the analyzing quadrupole increased to $\sim 3 \times 10^{-5}$ torr due to the increased gas load effusing through the interquad aperture. It was thought that the increased ion count rate of Figure 3 might somehow be an artifact of this higher pressure (due to ion feedback in the electron multiplier or some other unknown mechanism) and so an experiment was done in which the pressure in the rf rods was left at $2.4 \times 10^{-3}$ torr and the pressure in the analyzer region was increased from $5 \times 10^{-6}$ to $3 \times 10^{-5}$ torr (by partially closing the valve to the diffusion pump). No increases in ion signal were seen.

The increases in ion transmission then did not appear to be spurious artifacts but in fact a true improvement in transmission at the higher pressures. Figure 3 shows that the improvement is seen for $q$ values of 0.19 and 0.6 (and by implication values between 0.19 and 0.6 ) and is greater for heavier ions (al least for $m / z 832^{-}$in comparison with $m / z 196^{+}$ and $\left.391^{+}\right)$. The improved transmission seemed to imply the ions were confined closer to the center line of the quadrupole at the higher pressures. To investigate this further the experiments were repeated with a 1.0-mm (smaller) diameter interquad aperture.

Figure $4 \mathrm{a}$ and $\mathrm{b}$ compares the ion transmission through 1.0- and 2.5-mm diameter apertures for $\mathrm{m} / \mathrm{z}$ $196^{+}$and $m / z 391^{+}$ions $(q=0.19,10-\mathrm{eV}$ injection energy). In each case the ion count rates are normalized to 1.0 at a pressure of $2.4 \times 10^{-3}$ torr. Absolute ion signals were lower with the $1.0-\mathrm{mm}$ aperture. It is seen that the relative increase in count rate with pressure is greater with the smaller interquad aperture. This supports the hypothesis that the increased ion transmission derives from the ions being confined closer to the central axis at higher pressures. Collisions with the background gas apparently dampen or focus the ion trajectories on to the center line. Results
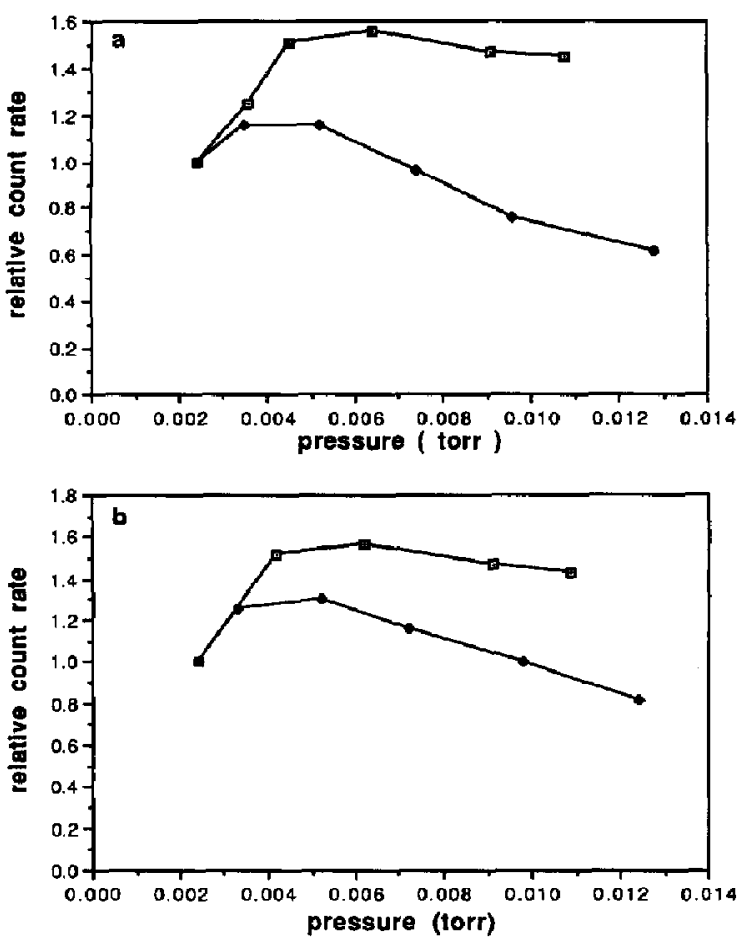

Figure 4. The variation of relative count rate for ions of (a) $m / z 196^{+}$and (b) $m / z 391^{+}$with pressure in the rf quadrupole with interquad apertures of $(\square)$ 1.0-mm diameter and $(\bullet) 2.5$ $\mathrm{mm}$ diameter. The results are normalized to 1.0 at a pressure of $2.4 \times 10^{-3}$ torr.

similar to those of Figures 3 and 4 have been found for $q$ values of 0.19 to 0.85 and ion injection energies of 5 to $30 \mathrm{eV}$.

The mass-resolved data give relative ion transmissions. To obtain the absolute transmission, measurements of the total ion current were done. With the corona source used for these measurements, the major ions were $\mathrm{H}_{3} \mathrm{O}^{+}, \mathrm{H}_{3} \mathrm{O}^{+} \cdot \mathrm{H}_{2} \mathrm{O}$ and $\mathrm{H}_{3} \mathrm{O}^{+} \cdot 2 \mathrm{H}_{2} \mathrm{O}$ with additional contributions from low molecular weight $(<100)$ chemical noise. The rf quadrupole was operated at a $\mathrm{q}$ of 0.6 for $\mathrm{m} / \mathrm{z} 35$ so that the $\mathrm{q}$ of ions ranged from 0.9 for $m / z 23$ (the low mass cut-off) to 0.21 for $m / z$ 100. The first measurements were done with a $2.5-\mathrm{mm}$ interquad aperture. The total current through the sampling orifice was measured to be $\mathbf{1 2 0}$ pA. The rod offset on the analyzing quadrupole was set to $+55 \mathrm{~V}$ (i.e., $30 \mathrm{~V}$ higher than the orifice) to prevent any ion transmission down the analyzing quadrupole. With a pressure of $6 \times 10^{-3}$ torr in the if rods region the total current to the interquad aperture plate was $100 \mathrm{pA}$, indicating greater than $80 \%$ transmission of ions down the rf rods (10-eV ion injection energy). The rod offset on the analyzing quadrupole was then lowered to $0 \mathrm{~V}$ and the current to the aperture plate was found to drop to $10 \mathrm{pA}$. This was 
interpreted as indicating that $90 \mathrm{pA}(-90 \%$ of all ions) were transmitted through the interquad aperture. This transmission is unexpectedly high because the $2.5-\mathrm{mm}$ aperture is only $-4 \%$ of the area of the inscribed circle of the rf quadrupole.

The current measurements were then repeated with a $1.0-\mathrm{mm}$ aperture. With the analyzing quadrupole rod offset set to $+55 \mathrm{~V}$ to prevent ion transmission through the aperture and an rf quadrupole pressure of $2.4 \times 10^{-3}$ torr, the total current to the aperture plate was $108 \mathrm{pA}$. With the analyzer rod offset lowered to $0 \mathrm{~V}$ the current dropped to $93 \mathrm{pA}$, indicating $15 \mathrm{pA}(\sim 15 \%)$ ion transmission. This current is $\sim 1 / 6$ of the current transmitted at this pressure by the 2.5-mm aperture, a ratio close to the ratio of the aperture areas (1/6.25). The experiment was repeated with the rf rods at $6 \times 10^{-3}$ torr. The total current to the interquad aperture with the analyzer rod offset 55 $\mathrm{V}$ was $75 \mathrm{pA}$, and with the analyzer rod offset $0 \mathrm{~V}$ it was $54 \mathrm{pA}$, indicating $21 \mathrm{pA}$ transmitted through the aperture, an increase of $\sim 40 \%$ over that at the lower pressure.

These current measurements show that the absolute ion transmission down the rf rods is high $(-80 \%)$ and that the absolute ion transmission through the interquad aperture can also be high, even though the aperture is small relative to the field diameter. The current measurements are an average for ions of $\mathrm{m} / \mathrm{z}$ 23 to $\sim$ 100. It is shown beluw (in Tables 2 and 3) (and is somewhat evident in Figures 3 and 4) that the increase in ion transmission at higher pressures is greater for higher mass ions; the implication is that with an interquad aperture as small as $1.0 \mathrm{~mm}$, nearly $100 \%$ ion transmission is possible for higher mass ions. The increase in relative analyte signal, the increase in total ion current, and the high absolute ion transmission at higher gas pressures in the rf rods are all interpreted as a collisional focusing effect where the ion trajectories are dampened and the ions are confined closer to the center line of the rf quadrupole. These collisional focusing effects were only observed at low $(1-30 \mathrm{eV})$ ion injection energies. At higher energies the absolute transmission of the $\mathrm{rf}$ quadrupole dropped and collisional focusing was not observed. Presumably at higher energies collisions are sufficiently energetic to scatter ions out of the quadrupole or possibly produce $\mathrm{CAD}$ of the ions (although CAD was not observed).

Table 2. Ratio of count rates obtained at $5 \times 10^{-3}$ torr to those at $5 \times 10^{-4}$ torr

\begin{tabular}{lrcc}
\hline Compound & Mass & $M / 2$ & Ratio \\
\hline \hline DMMPA & 196 & 196 & 7.1 \\
PPG & 906 & 906 & 8.6 \\
Mellitin & 2845 & 712 & 15 \\
Insulin & 5740 & 1144 & 40 \\
Myoglobin & 16.950 & 893 & 79 \\
\hline
\end{tabular}

Table 3. Ratio of count rates obtained at $5 \times 10^{-3}$ torr to those at $5 \times 10^{-4}$ torr with the ion injection energy oplimized at each pressure

\begin{tabular}{lrrr}
\hline Compound & Mass & $M / z$ & Ratio \\
\hline \hline DMMPA & 196 & 196 & 3.9 \\
PPG & 906 & 906 & 6.9 \\
Myoglobin & 16,950 & 893 & 10.9 \\
\hline
\end{tabular}

\section{Results with the Differentially Pumped Interface}

Experiments were then done with the differentially pumped interface of Figure 2. As mentioned, this interface allowed operation of the rf quadrupole at lower pressures $\left(-5 \times 10^{-4}\right.$ torr). At the same time the ionspray source was added to the system, allowing investigation of higher mass compounds. With this interface the spacing between the skimmer tip and $\mathrm{rf}$ rods $(45 \mathrm{~mm})$ was relatively large. It was found that the optimum voltage difference (i.e., for maximum ion transmission) between the skimmer and $\mathrm{rf}$ quadrupole (ion injection energy) depended on the pressure in the quadrupole. At low pressures $(-\leq 5$ $\times 10^{-4}$ torr) $\sim 80 \mathrm{~V}$ was optimum; at high pressures $\left(>2 \times 10^{-3}\right.$ torr $)-10 \mathrm{~V}$ was optimum. At Jow pressures, i.e., in the absence of collisions, the $80 \mathrm{~V}$ difference is presumably preferred to inject ions optimally for the acceptance of the quadrupole. At high pressures low energies are preferred to optimize collisional focusing in the rf quadrupole (at the expense of ion injection efficiency).

Figure 5 shows the relative count rate as a function of pressure for myoglobin +13 ions $(m / z=1304$ ) (ion injection energy $10 \mathrm{eV}, \mathrm{q}=0.19$ ). The relative count rate is normalized to 1.0 at a pressure of $5 \times 10^{-4}$ torr. The gain in transmission at high pressure is dramatic, $\sim \times 70$ ! It is also evident that considerable collisional focusing takes place even at $2.5 \times 10^{-3}$

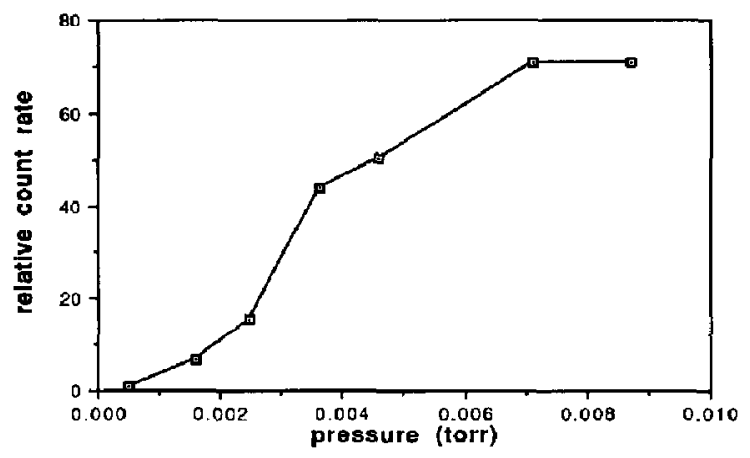

Figure 5. The variation of relative count rate for ions of $\mathrm{m} / \mathrm{z}$ $1304(+13)$ from myoglobin with pressure in the rf quadrupole. The results are normalized to 1.0 at a pressure of $5.0 \times 10^{-4}$ torr. 
torr, the lowest pressure possible with the single aperture interface.

The experiment of Figure 5 was repeated with ions from DMMPA $\left(m / z 196^{+}\right)$, PPG $\left(m / z 906^{+}\right)$, mellitin $+4\left(\mathrm{~m} / z 712^{+}\right)$, insulin $+5\left(\mathrm{~m} / z 1144^{+}\right)$, and myoglobin $+19\left(\mathrm{~m} / z 893^{+}\right)$. The ion injection voltage was set for optimum ion transmission at a pressure of $5 \times 10^{-3}$ torr. The results are summarized in Table 2 , which shows the ratio of ion count rates obtained at $5 \times 10^{-3}$ torr to those at $5 \times 10^{-4}$ torr. The improvements in ion transmission are considerably greater than those of Figures 3 and 4 (where the results are normalized at $2.4 \times 10^{-3}$ torr). In addition, the increase in signal is seen to be greater for the higher mass compounds. Note it is the mass of the compound (m), not the mass-to-charge ratio, that determines the extent of collisional focusing. For example, mellitin +4 and insulin +19 are similar in mass-tocharge ratio but very different in mass; insulin shows a significantly greater collisional focusing effect. This mass effect provides additional evidence that it is collisions with the background gas which provide the damping of the ion trajectories because it is the ratio of the mass of the ion to the collision gas mass that determines the kinematics of the collisions.

The results of Table 2 somewhat exaggerate the practical improvements that can be gained from collisional focusing because, as discussed, the ion transmission optimized at different ion injection energics at high and low pressures. The experiment was repeated with the ion injection energy adjusted for best transmission at both low pressure $\left(5 \times 10^{-4}\right.$ torr $)$ then at high pressure $\left(5 \times 10^{-3}\right.$ torr). The results are summarized in Table 3. The enhancements of Table 3 are significantly less than those of Table 2, but still show an increase with mass (not mass-to-charge ratio) and an increase of an order of magnitude in ion transmission for myoglobin.

\section{Ion Energy Effects}

All of the collisional focusing effects discussed so far were obtained with the interquad aperture and analyzing quadrupole rod offset set to a voltage lower than the rod offset of the if quadrupole. In the course of this work it was found that if the interquad aperture had a potential higher than the rf rod offset (but still lower than the nominal ion injection energy), no increases in transmission with pressure were observed and, in fact, substantial losses in ion transmission were encountered. An example is shown in Figure 6. The data were obtained with the single aperture interface and a nominal ion injection energy of $15 \mathrm{eV}$. It is seen that for an interquad aperture potential even $1 \mathrm{~V}$ higher than the rf quadrupole offset, substantial losses in transmission occur at higher pressure. This initially puzzling effect led us to investigate the kinetic energies of ions exiting the if quadrupole.

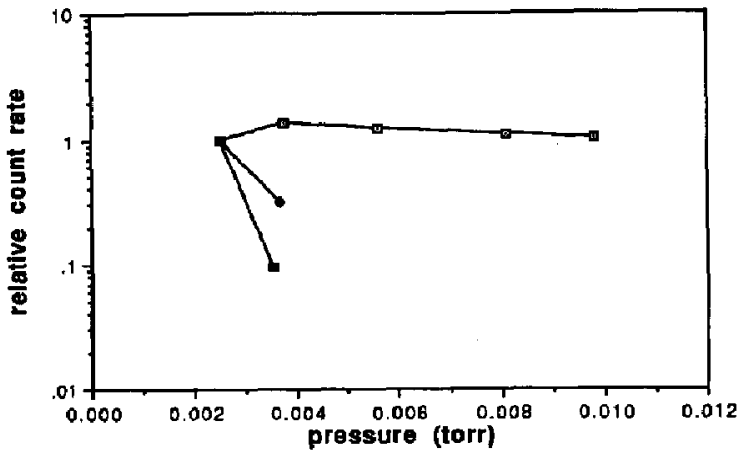

Figure 6. The variation of relative count rate for ions of $m / z$ $391^{+}$with pressure in the if quadrupole for three values of the voltage on the interquad aperture above that of the if quadrupole rod offset. $\square=0 \mathrm{~V} ;=1.0 \mathrm{~V} ; \square=10 \mathrm{~V}$. The nominal ion injection energy was $15 \mathrm{eV}$.

Ion energies were measured approximately by using the analyzing quadrupole rod offset to generate a stopping curve. Figures 7-9 show stopping curves for $m / z 196^{+}, 391^{+}$, and 832 at three pressures in the $2.4 \times 10^{-3}$ to $1.0 \times 10^{-2}$ torr range. In Figures 7-9 the "stopping" voltage is the rod offset of the analyzer above the rod offset of the rf quadrupole. In all cases the relative count rate is normalized to 1.0 at a stopping potential of $0 \mathrm{~V}$. It is immediately obvious that the stopping curves change dramatically with pressure and that the ions have much less energy than the nominal $25-\mathrm{eV}$ injection energy. At the higher pressures the ion energies are considerably moderated by collisions with the background gas. For example, at $5.9 \times 10^{-3}$ torr only about $10^{-4}$ of $\mathrm{m} / \mathrm{z} 196$ ions have more than $4 \mathrm{eV}$ axial energy (Figure 7). It is also seen that the heavier ions are slowed less than the lighter ions, as might be expected. It is clear why potentials downstream of the rf quadrupole must be kept lower than the offset of the quadrupole. Ions are slowed by collisions with the background gas to the point where they have only a few electron volts or

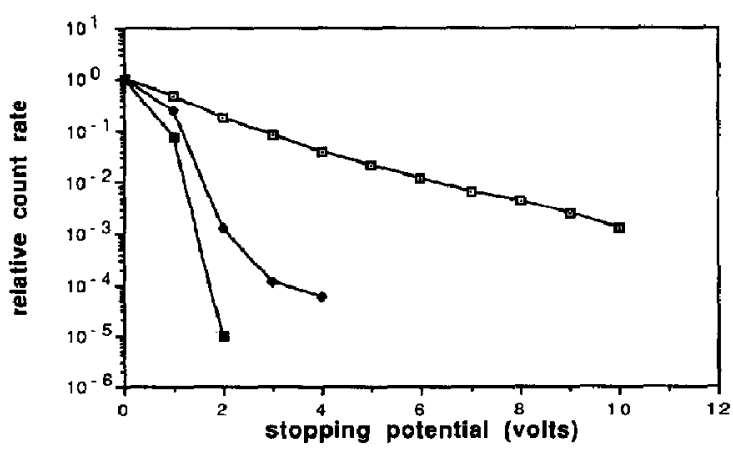

Figure 7. Stopping curves for ions of $m / z 196^{+}$for pressures in the If quadrupole of ( $\square$ ) $2.4 \times 10^{-3}$ torr, ( $) 5.9 \times 10^{-3}$ torr, and (⿴) $9.8 \times 10^{-3}$ torr. 


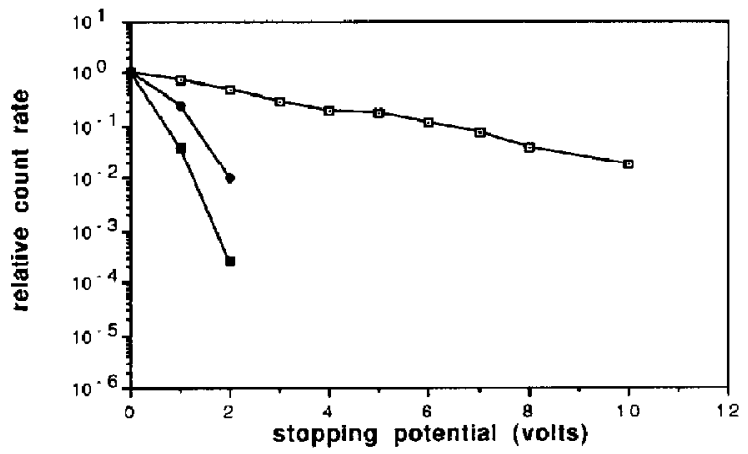

Tigure 8. Stopping curves for ions of $m / z 391^{+}$for pressurcs in the rf quadrupole of $(\square) 2.4 \times 10^{-3}$ torr, ( $\square$ ) $5.9 \times 10^{-3}$ torr, and (a) $9.8 \times 10^{-3}$ torr.

less axial energy. These ions cannot surmount any appreciable potential barriers after exiting the if quadrupole.

\section{Monte Carlo Models}

Simple Monte Carlo models were developed to simulate the energy loss process for ions passing through the relatively high pressure quadrupole. (A discussion of the use of Monte Carlo models for energy loss calculations is given in ref $\mathbf{1 3}$ and an application to ion motion in gases in ref 14.) Two models were developed here. The first simulates ions passing through a cell uniformly filled with gas (applicable to the apparatus of Figure 2). The second simulates ions accelerated through a free jet and then through a region uniformly filled with gas (applicable to the apparatus of Figure 1, D. J. Douglas, unpublished). The first (simpler) model is briefly described here to illustrate the principle of the method.

The Monte Carlo method models the motion of ions in a collision cell by statistically calculating a distance traveled before an ion has a first collision. The scattering angle and hence energy of the ion after

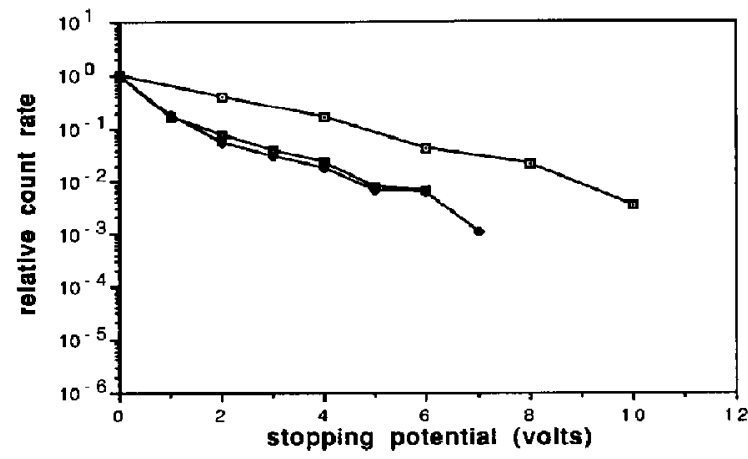

Figure 9. Stopping curves for ions of $m / z 832^{-}$for pressures in the rf quadrupole of (口) $2.5 \times 10^{-3}$ torr, (\$) $5.6 \times 10^{-3}$ torr, and (D) $8.6 \times 10^{-3}$ torr. the first collision is calculated from the impact parameter of the collision (selected statistically). A new distance traveled is calculated and the process is repeated until the total distance traveled equals or exceeds the collision cell length (in this case the length of the rf quadrupole). The entire calculation is then repeated for many ions and a histogram of final ion energies is built up to give the energy distribution of ions exiting the collision cell.

The probability $(\overline{\mathrm{P}})$ of an ion not having a collision in a distance $l$ in a region of gas number density $n$ is

$$
\overline{\mathrm{P}}(l)=\mathrm{e}^{-\sigma \mathrm{n} l}
$$

where $\sigma$ is the collision cross-section. The probability of having a collision is $\mathrm{P}(l)=1-\overline{\mathrm{P}}(l)$ or

$$
\mathrm{P}(l)=1-\mathrm{e}^{-\mathrm{on} l}
$$

A random number $0 \leq \xi \leq 1$ was generated and eq 2 solved for $l$ with $\mathrm{P}(l)=\xi$. At this point the ion was considered to have a collision with energy $E$. (E equals the injection energy if this is the furst collision.) The outcome of the collision depends on the scattering angle in center-of-mass coordinates $\left(\theta_{\mathrm{cm}}\right)$, which in furn is a function of the impart parameter. The impact parameter $b$ has a probability distribution.

$$
\mathrm{P}(\mathrm{b}) \sim \mathrm{b} d \mathrm{~b}
$$

The impact parameter was calculated by picking two random numbers $\xi_{1}$ or $\xi_{2}$ and taking

$$
\begin{array}{ll}
\mathrm{b}=\xi_{1} \mathrm{~b}_{\max } & \text { if } \xi_{1}>\xi_{2} \\
\mathrm{~b}=\xi_{2} \mathrm{~b}_{\max } & \text { if } \xi_{2}>\xi_{1}
\end{array}
$$

where $b_{\max }$ is the maximum impact parameter. The collision cross-section $\sigma$ is given by

$$
\sigma=\pi \mathrm{b}_{\max }^{2}
$$

For a hard sphere collision the scattering angle in center-of-mass coordinates is given by [15]

$$
\theta_{\mathrm{cm}}=2 \arccos \left(\mathrm{b} / \mathrm{b}_{\max }\right)
$$

and the kinetic energy after the collision in the LAB frame $\left(E^{\prime}\right)$ is given by [16]

$$
\frac{E^{\prime}}{E}=\frac{m_{1}^{2}+m_{2}^{2}}{M^{2}}+\frac{2 m_{1} m_{2}}{M^{2}}\left(\cos \theta_{c m}\right)
$$

where $m_{1}$ is the mass of the ion, $m_{2}$ the mass of the target, and $M=m_{1}+m_{2}$. The kinetic energy of the ion after each collision is calculated from eq 7 , a new scattering length is calculated, and the process is repeated. Typically 1000 ions were used to generate a histogram of the final ion energies. The model is very simple because it does not include (at all) the effect of 
the quadrupole field on the ion trajectories and does not consider the effect of angular scattering on the total path length. The hard sphere potential used might be questioned, but because each ion has many collisions, and because the energy distributions are an average over many ions, the results are insensitive to the potential.

Calculated energy distributions for ions of mass $196\left(\mathrm{E}_{\mathrm{o}}=25 \mathrm{eV}\right)$ passing through a $15-\mathrm{cm}$ cell at pressures of $1 \times 10^{-4}, 5 \times 10^{-4}, 1 \times 10^{-3}, 2 \times 10^{-3}$, and $5 \times 10^{-3}$ torr are shown in Figure $10 \mathrm{a}$ to $e$, respectively. A scattering cross-section of $50 \AA^{2}\left(b_{\max }\right.$ $=4.0 \AA$ ) was used. It is seen that for reasonable

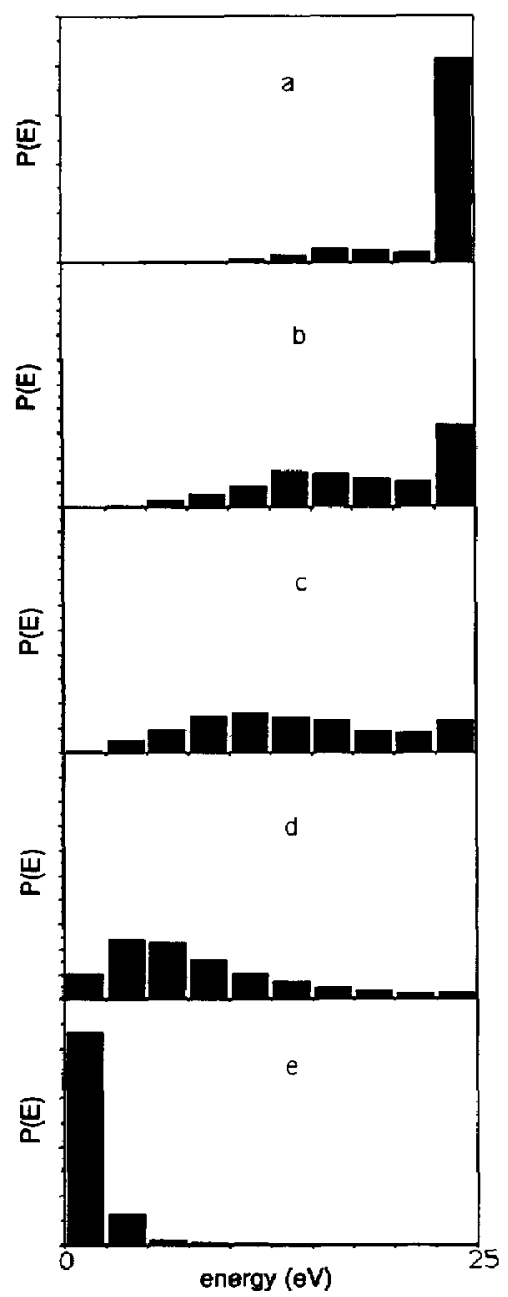

Figure 10. Calculated energy distributions for ions of $\mathrm{m} / \mathrm{z}$ $196^{+}$at pressures of the if quadrupole of (a) $1.0 \times 10^{-4}$ torr, (b) $5.0 \times 10^{-4}$ torr, (c) $1.0 \times 10^{-3}$ torr, (d) $2.0 \times 10^{-3}$ torr, and (e) $5.0 \times 10^{-3}$ torr. An injection energy of $25 \mathrm{eV}$ and maximum impact parameter of $4.0 \AA$ were used in the calculation. The energy scale ranges from $0 \mathrm{eV}$ on the left to $25 \mathrm{eV}$ on the right and is divided into ten "bins" of $2.5 \mathrm{eV}$ width. The vertical axis ranges from 0 to 1.0 in each part. cross-sections the model predicts that the ions can lose a substantial fraction of their initial energy, as is observed.

Results from the second model, describing acceleration of ions through a free jet in the rf quadrupole followed by loss of energy in the quadrupole, are shown in Figure 11. Ions of mass 391 were modeled with a $25-\mathrm{V}$ potential applied between the orifice and rf quadrupole entrance. A collision cross-section of $102 \AA^{2}\left(b_{\max }=5.7 \AA\right)$ was used. Figure 11a shows the distribution emerging from the $\mathrm{rf}$ quadrupole when the pressure is $1 \times 10^{-5}$ torr and the effect of collisions in the quadrupole is negligible. This is the distribution formed by accelerating the ions through the free jet. It is seen that ions lose $\sim 5 \mathrm{eV}$ of the 25 $\mathrm{eV}$ energy they would have if there were no collisions in the jet. Distributions calculated from this model are in fair agreement with distributions calculated from the Boltzmann equation [17]. Figure 11b and $c$ shows

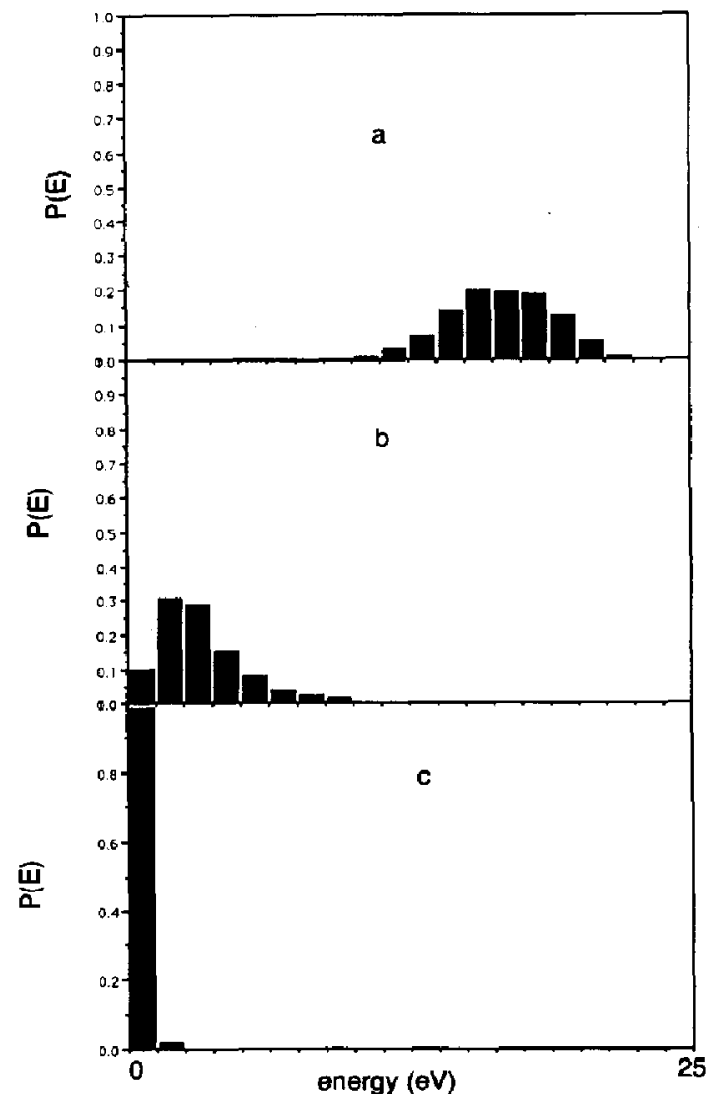

Figure 11. Calculated energy distributions for ions of $\mathrm{m} / \mathrm{z}$ $391^{+}$at pressures of the rf quadrupole of (a) $1.0 \times 10^{-5}$ torr, (b) $2.5 \times 10^{-3}$ torr, and (c) $5.9 \times 10^{-3}$ torr. A potential difference of $25 \mathrm{~V}$ between the orifice and rf quadrupole offset was used. The maximum impact parameter was $5.7 \AA$. The energy scale ranges from $0 \mathrm{eV}$ on the left to $25 \mathrm{eV}$ on the right and is divided into twenty "bins" of $1.25 \mathrm{eV}$ width. The vertical axis ranges from 0 to 1.0 in each part. 
the modification of the initial distribution of Figure 11 a by collisions in the quadrupole at pressures of $2.5 \times 10^{-3}$ and $5.9 \times 10^{-3}$ torr, respectively. Again, substantial losses in ion energy are seen.

Figure 12 shows stopping curves for $\mathrm{m} / \mathrm{z} 391^{+}$ derived from the calculated energy distributions of Figure $11 \mathrm{~b}$ and $\mathrm{c}$ and compares these directly to the experimental data taken from Figure 8. For the crosssection used $\left(102 \AA^{2}\right)$ the model matches the experimental results fairly well, given the limitations of the model and of the stopping curves generated with the quadrupole rod offset. These model calculations then show that appreciable energy loss can be expected for ions transmitted through an rf quadrupole in the $10^{-3}$ torr range. "Reasonable" collision cross-sections can provide fair agreement with the experimental energy distributions and the variation of these distributions with pressure. The mean free path $(\lambda)$ for an ion can be taken as

$$
\lambda=1 / \mathbf{n} \sigma
$$

For a collision cross-section of $102 \AA^{2}$ and an rf quadrupole pressure of $5.9 \times 10^{-3}$ torr, this is calculated to be $0.50 \mathrm{~cm}$. An ion traveling the length of the 15-cm quadrupole then has -30 collisions. This evidently is enough to provide collisional focusing for an ion of mass $391 \mathrm{u}$.

\section{Extension to Higher Pressure}

It is of interest to ask how high the pressure can be in the rf quadrupole before ion transmission is substantially reduced. All the experiments discussed so far were carried out at pressures of $\sim 1 \times 10^{-3}$ to $2 \times$ $10^{-2}$ torr because this is where the ion transmission maximized. Figure 13 shows the transmission of $\mathrm{m} / \mathrm{z}$ $196^{+}$and $\mathrm{m} / \mathrm{z} 391^{+}$at pressures up to $6.5 \times 10^{-2}$ torr (normalized to the transmission at $2.4 \times 10^{-3}$

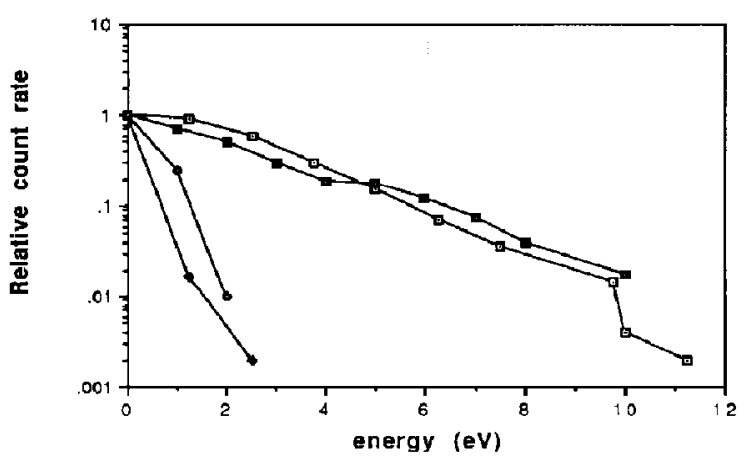

Figure 12. Stopping curves calculated from the energy distribution of Figure 11 compared with the experimental curves of Figure 8 as follows: $\square=$ model calculation at $2.5 \times 10^{-3}$ torr; $\mathbf{\square}=$ experimental result at $2.5 \times 10^{-3}$ torr; $\$=$ model calculation at $5.9 \times 10^{-3}$ torr; $\diamond=$ experimental result at $5.9 \times 10^{-3}$ torr.

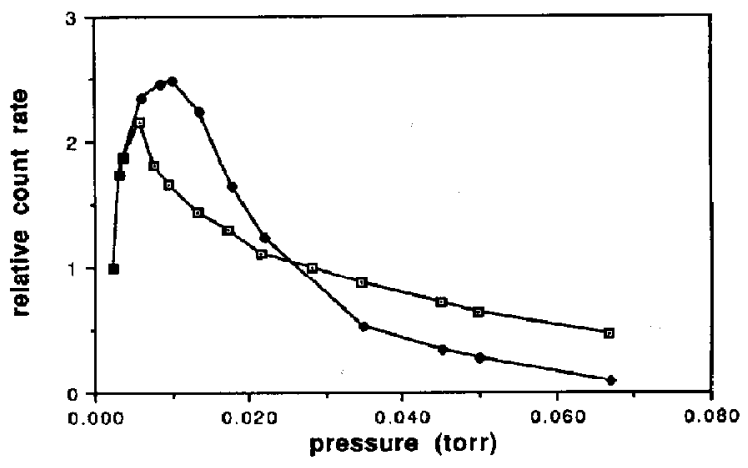

Figure 13. The variation of count rate for ions of $(\square) m / z 196^{+}$ and $(\$) 391^{+}$with pressure in the rf rods from $2.4 \times 10^{-3}$ to $6.5 \times 10^{-2}$ torr $(q=0.19)$.

torr). The transmission decreases somewhat but remains appreciable and is similar to that expected at a pressure of $5 \times 10^{-4}$ torr. Remarkably, the highest pressures of Figure 13 are two to three orders of magnitude higher than those that have been used in the past in systems incorporating $\mathrm{rf}$ quadrupoles to sample ions from "gassy" sources. Some of the loss in transmission at the highest pressure seen in Figure 13 may be due to changes in the ion energy distribution. At $6 \times 10^{-2}$ torr the ions are expected to have extremely low energies, which may affect their transmission through the interquad aperture and fringing fields at the entrance to the mass analyzing quadrupole.

\section{Discussion}

The increased transmission through the rf quadrupole at higher pressures appears to be caused by collisional damping of the ion trajectories, which confines ions closer to the center line. At the same time the ions lose a substantial fraction of their axial energy. The result appears to be analogous to the behavior of ions in three-dimensional ion traps where collisions remove radial energy, leaving the ions closer to the trap center. In the three-dimensional trap the motion of ions in the $z$ direction can be thought of as motion in a potential well of depth $(\bar{D})$ given by $[5,7]$

$$
\overline{\mathrm{D}}=\frac{1}{8} \mathrm{q}_{z} \mathrm{~V}
$$

where $\mathrm{q}_{z}$ is the Mathieu parameter for motion in the $\mathrm{z}$ direction and $\mathrm{V}$ is the zero to peak of voltage applied between the ring and end cap electrodes. Loss of energy in collisions causes ions to move to the center of the trap where the effective potential is lowest and thus the loss of ion energy is an important part of the mechanism of collisional focusing. While simplistic, because it ignores the detailed dynamics of the ion motion, this interpretation can be applied equally to 
the two-dimensional rf quadrupole where $q_{z}$ of eq 9 can be replaced by $\mathrm{q}_{x}$ or $\mathrm{q}_{y}$ for transverse motion in the rf quadrupole. By analogy then, energy loss is expected to be an important part of the mechanism of collisional focusing in the linear $\mathrm{rf}$ quadrupole. The energy distributions and stopping curves reported here show directly a loss of axial energy. Losses of radial energy can only be inferred.

The results reported here show collisional focusing of ions with masses from 196 to 16,950 in collisions with nitrogen. In ather tests we have found collisional focusing for ions as low as $m / z \mathbf{4 6}$. However, $m / z 19$ showed no focusing. It is believed that the collisional focusing will be effective for all ions of mass greater than the target mass. In this case collisions will always leave ions with some forward momentum, allowing them to exit the quadrupole. Ions lighter than the collision gas, however, can be backscattered and essentially stopped in the quadrupole. It has been argued [18] that in three-dimensional traps ions lighter than the collision gas will show an exponential increase in energy and eventually be lost. This mechanism may apply here. The results reported here also show improved collisional focusing for higher mass ions. This may be due to a combination of effects as follows: (1) I ligher mass ions, while having the same $\mathrm{q}$ in the rf quadrupole, are transmitted with a higher absolute voltage on the rods and so, by eq 9, they have a grealer well depth with better confinement. (2) Higher mass ions have a smaller fraction of their kinetic energy transferred to radial energy in a collision and hence may be less likely to scatter out of the quadrupole.

It is at first surprising that an ion of $m / z 16,950$ (myoglobin) should show any collision effects with nitrogen. Because of the very large mass difference, a collision does very little to perturb the trajectory of such a massive ion. For example, eq 7 shows that at a scattering angle $\left(\theta_{\mathrm{cm}}\right)$ of $90^{\circ}$, a myoglobin ion will lose only $\sim 0.3 \%$ of its kinetic energy. It was argued above that for collisional focusing to occur, an ion must lose an appreciable fraction of its radial kinetic energy. For a moderate mass ion $\left(m / z 391^{+}\right)$some tens of collisions are sufficient to achieve this (see Figure 12). For an ion like myoglobin several hundred collisions are necessary. (Monte Carlo simulation verifies this.) Because collisional focusing is seen for such ions, collision cross-sections must be $-1000 \AA^{2}$ or more for these ions. It would be of interest to measure stopping curves for a variety of peptide or protein ions. Matching the experimental data with the Monte Carlo model could give "model" cross-sections for these ions that may be useful in revealing novel conformational changes for different charge states.

Despite the widespread use of $\mathrm{rf}$ quadrupoles for transport [1-3] and CAD of organic (see, e.g., ref 19) and inorganic ions [20-22], collisional focusing, to our knowledge, has not been reported previously. To some extent this may be because the conditions opti- mum for observing collisional focusing (low ion axial energy and a small exit aperture) are not favorable when rf quadrupoles are used as CAD cells. In this case higher ion energies and the largest possible exit aperture are preferred $[4,16,19]$ to increase fragment ion yield and transmission. Under these conditions only scattering losses are normally observed.

Operation of $\mathbf{r f}$ quadrupoles in this collisional focusing regime may offer a practical advantage for sampling from API sources. With the apparatus of Figure 1 or Figure 2, the pressure in the rf quadrupole chamber can be $\sim \times 10$ (or more) higher than has previously been used. This means a pump of lower speed $(\sim \times 10)$ can be used with cost, size, and weight savings. Conversely, for a given pump speed, the gas flow can be increased $\sim \times 10$ and, hence, for a given ion-to-gas ratio [23] entering the rf quadrupole the sensitivity can be improved by the same factor. In both cases a narrowly confined ion beam (approximately a few millimeters) with low energy spread (a few electrovolts) is delivered to the mass analyzing quadrupole. The reduction in ion axial energy spread may help to improve the mass resolution of the analyzing quadrupole, at least in those cases where the source produces a large energy spread (e.g., Figure 11).

\section{Acknowledgment}

This work was partially supported by the Ontario Technology Fund.

\section{References}

I. Dawson, P. H.; French, J. B.; Huckley, J. A.; Douglas, D. J.; Simmons, D. Org. Mass Spectrometry 1982, 17, 205.

2. Smith, R. D.; Olivares, J. A.; Nguyen, N. T.; Udseth, H. R. Anal. Chem. 1988, 60, 436.

3. Henion, J.; Wachs, T. Procedtings of the 39th ASMS Conference on Mass Spectrometry and Allied Topics; Nashville, TN, 1991; Paper ThRP30.

4. Dawson, P. H., Fulford, J. E. Int. I. Mass Spectrom. Ion Phys. 1982, 42, 195.

5. Dehmelt. H. G. Advances At. Mol. Phys. 1967, 3, 53.

6. Stafford, G. C.; Kelley, P. E.; Syka, J. E. P.; Reynolds, W. E.; Todd, J. F. J. Int. J. Mass Spec. Ion Proc. 1984, 60, 85.

7. March, R. E.; Hughes, R. J. Quadrupole Storage Mass Spectrometry; John Wiley: New York, 1989; PP 151-154.

8. Bruins, A. P.; Covey, T. R.; Henion, J. D. Anal. Chem. 1987, $59,2642$.

9. Dawson, P. H. Quadrupole Mass Spectrometry and its Applications; Elsevier Scientific, Amsterdam, 1976.

10. Smith, R. D.; Loo, J. A.; Barinaga, C. G. E.; Udseth, H. R. J. Am. Sac. Mass Spectrom. 1990, 1, 53.

11. Chowdluury, S. K.; Viswanatham, K., Chail, B. T. Rapid Comm. Mass Spec. 1990, 4, 81.

12. Covey, T. R; Bonner, R. F.; Shushan, B. I. Rapid Com. Mass Spec. 1988, 2, 249.

13. Ziegler, J. F.; Biersack, J. P.; Littmark, U. The Stopping and Range of Ions In Solids; Pergamon, New York, 1985.

14. Skullerud, H. R. J. Phys. B. Atom. Molec. Phys. 1973, 6, 728. 
15. Hirschfelder, J. O.; Curtiss, C. F.; Bird, R, B, Molecular Theory of Gases and Liquids; Wiley: New York, 1954.

16. See, for example, Douglas, D. J. J. Phys. Chem. 1982, 86, 185 (eq 7 with $E_{\text {int }}=0$ ).

17. Darcie, T. E., University of Toronto Institute for Aerospace Studies, 1983, Report \#269.

18. Mayor, F. G.; Dehmelt, H. G. Phys, Rev, 1968, $170,91$.
19. Dawson, P, H.; Douglas, D. J. In Tandem Mass Spectrometry, McLafferty, F. W., Ed.; John Wiley: New York, 1983; p 125. 20. Rowan, J. T.; I louk, R. S. Applied Spectrose. 1989, 43, 976. 21. Douglas, D. J. Can J. Spectrosc. 1989, 34, 38.

22. Duckworth, D. C.; Marcus, R. K. Applied Spectrosc. 1990, 44, 649.

23. Bruins, A. Mass Spectrometry Reviews 1991, 10, 53. 